\title{
Search for TeV Gamma-ray Emission from GRB 100621A, an extremely bright GRB in X-rays, with H.E.S.S.
}

H.E.S.S. Collaboration, A. Abramowski ${ }^{1}$, F. Aharonian ${ }^{2,3,4}$, F. Ait Benkhali ${ }^{2}$, A. G. Akhperjanian ${ }^{5,4}$, E. Angüner ${ }^{6}$, G. Anton ${ }^{7}$, S. Balenderan ${ }^{8}$, A. Balzer ${ }^{9}, 10$, A. Barnacka ${ }^{11}$, Y. Becherini ${ }^{12}$, J. Becker Tjus ${ }^{13}$, K. Bernlöhr ${ }^{2,6}$, E. Birsin ${ }^{6}$, E. Bissaldi ${ }^{14}$, J. Biteau ${ }^{15}$, M. Böttcher ${ }^{16}$, C. Boisson ${ }^{17}$, J. Bolmont ${ }^{18}$, P. Bordas ${ }^{19}$, J. Brucker ${ }^{7}$, F. Brun ${ }^{2}$, P. Brun ${ }^{20}$, T. Bulik ${ }^{21}$, S. Carrigan ${ }^{2}$, S. Casanova $^{16,2}$, M. Cerruti ${ }^{17,22}$, P. M. Chadwick ${ }^{8}$, R. Chalme-Calvet ${ }^{18}$, R.C.G. Chaves ${ }^{20,2}$, A. Cheesebrough ${ }^{8}$, M. Chrétien ${ }^{18}$, S. Colafrancesco ${ }^{23}$, G. Cologna $^{12}$, J. Conrad $^{24}$, C. Couturier ${ }^{18}$, M. Dalton ${ }^{25,26}$,

M. K. Daniel ${ }^{8}$, I. D. Davids ${ }^{27}$, B. Degrange ${ }^{15}$, C. Deil ${ }^{2}$, P. deWilt ${ }^{28}$, H. J. Dickinson ${ }^{24}$, A. Djannati-Ataï ${ }^{29}$, W. Domainko ${ }^{2}$, L. O'C. Drury ${ }^{3}$, G. Dubus ${ }^{30}$, K. Dutson ${ }^{31}$, J. Dyks ${ }^{11}$, M. Dyrda ${ }^{32}$, T. Edwards ${ }^{2}$, K. Egberts ${ }^{14}$, P. Eger ${ }^{2}$, P. Espigat ${ }^{29}$, C. Farnier ${ }^{24}$, S. Fegan ${ }^{15}$, F. Feinstein ${ }^{33}$, M. V. Fernandes ${ }^{1}$, D. Fernandez ${ }^{33}$, A. Fiasson ${ }^{34}$, G. Fontaine ${ }^{15}$, A. Förster ${ }^{2}$, M. Füßling ${ }^{10}$, M. Gajdus ${ }^{6}$, Y. A. Gallant ${ }^{33}$, T. Garrigoux ${ }^{18}$, B. Giebels ${ }^{15}$, J. F. Glicenstein ${ }^{20}$, M.-H. Grondin ${ }^{2,12}$, M. Grudzińska ${ }^{21}$, S. Häffner ${ }^{7}$, J. Hahn ${ }^{2}$, J. Harris ${ }^{8}$, G. Heinzelmann ${ }^{1}$, G. Henri ${ }^{30}$, G. Hermann ${ }^{2}$, O. Hervet ${ }^{17}$, A. Hillert ${ }^{2}$, J. A. Hinton ${ }^{31}$, W. Hofmann ${ }^{2}$, P. Hofverberg ${ }^{2}$, M. Holler ${ }^{10}$, D. Horns ${ }^{1}$, A. Jacholkowska ${ }^{18}$, C. Jahn ${ }^{7}$, M. Jamrozy ${ }^{35}$, M. Janiak ${ }^{11}$, F. Jankowsky ${ }^{12}$, I. Jung ${ }^{7}$, M. A. Kastendieck ${ }^{1}$, K. Katarzyński ${ }^{36}$, U. Katz ${ }^{7}$, S. Kaufmann ${ }^{12}$, B. Khélifi ${ }^{15}$, M. Kieffer ${ }^{18}$, S. Klepser ${ }^{9}$, D. Klochkov ${ }^{19}$, W. Kluźniak ${ }^{11}$, T. Kneiske ${ }^{1}$, D. Kolitzus ${ }^{14}$, Nu. Komin ${ }^{34}$, K. Kosack ${ }^{20}$, S. Krakau ${ }^{13}$, F. Krayzel ${ }^{34}$, P. P. Krüger ${ }^{16,2}$, H. Laffon ${ }^{25}$, G. Lamanna ${ }^{34}$, J. Lefaucheur ${ }^{29}$, A. Lemière ${ }^{29}$, M. Lemoine-Goumard ${ }^{25}$, J.-P. Lenain ${ }^{18}$, D. Lennarz ${ }^{2, \star}$, T. Lohse ${ }^{6}$, A. Lopatin ${ }^{7}$, C.-C. Lu ${ }^{2}$, V. Marandon ${ }^{2}$, A. Marcowith ${ }^{33}$, R. Marx ${ }^{2}$, G. Maurin ${ }^{34}$, N. Maxted ${ }^{28}$, M. Mayer ${ }^{10}$, T. J. L. McComb ${ }^{8}$, J. Méhault ${ }^{25,26}$, U. Menzler ${ }^{13}$, M. Meyer ${ }^{1}$, R. Moderski ${ }^{11}$, M. Mohamed ${ }^{12}$, E. Moulin ${ }^{20}$, T. Murach ${ }^{6}$, C. L. Naumann ${ }^{18}$,

M. de Naurois ${ }^{15}$, J. Niemiec ${ }^{32}$, S. J. Nolan ${ }^{8}$, L. Oakes ${ }^{6}$, P. T. O’Brien ${ }^{37}$, S. Ohm ${ }^{31,37}$, E. de Oña Wilhelmi ${ }^{2}$, B. Opitz ${ }^{1}$, M. Ostrowski ${ }^{35}$, I. Oya ${ }^{6}$, M. Panter ${ }^{2}$, R. D. Parsons ${ }^{2}$, M. Paz Arribas ${ }^{6}$, N. W. Pekeur ${ }^{16}$, G. Pelletier $^{30}$, J. Perez $^{14}$, P.-O. Petrucci ${ }^{30}$, B. Peyaud ${ }^{20}$, S. Pita ${ }^{29}$, H. Poon ${ }^{2}$, G. Pühlhofer ${ }^{19}$, M. Punch ${ }^{29}$, A. Quirrenbach ${ }^{12}$, S. Raab ${ }^{7}$, M. Raue ${ }^{1}$,

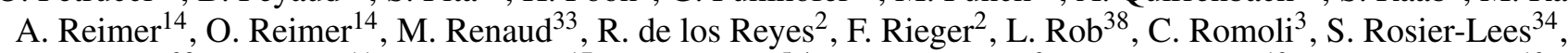

G. Rowell $^{28}$, B. Rudak ${ }^{11}$, C. B. Rulten ${ }^{17}$, V. Sahakian ${ }^{5,4}$, D. A. Sanchez ${ }^{2}$, A. Santangelo ${ }^{19}$, R. Schlickeiser ${ }^{13}$,

F. Schüssler ${ }^{20}$, A. Schulz ${ }^{9}$, U. Schwanke ${ }^{6}$, S. Schwarzburg ${ }^{19}$, S. Schwemmer ${ }^{12}$, H. Sol $^{17}$, G. Spengler ${ }^{6}$, F. Spies ${ }^{1}$, Ł. Stawarz ${ }^{35}$, R. Steenkamp 27 , C. Stegmann ${ }^{10,9}$, F. Stinzing 7 , K. Stycz ${ }^{9}$, I. Sushch ${ }^{6,16}$, A. Szostek ${ }^{35}$, P. H. T. Tam ${ }^{39}$, J.-P. Tavernet ${ }^{18}$, T. Tavernier ${ }^{29}$, A. M. Taylor ${ }^{3}$, R. Terrier $^{29}$, M. Tluczykont ${ }^{1}$, C. Trichard ${ }^{34}$, K. Valerius ${ }^{7}$, C. van Eldik ${ }^{7}$, G. Vasileiadis ${ }^{33}$, C. Venter ${ }^{16}$, A. Viana ${ }^{2}$, P. Vincent ${ }^{18}$, H. J. Völk ${ }^{2}$, F. Volpe ${ }^{2}$, M. Vorster ${ }^{16}$, S. J. Wagner ${ }^{12}$, P. Wagner ${ }^{6}$, M. Ward ${ }^{8}$, M. Weidinger ${ }^{13}$, Q. Weitzel ${ }^{2}$, R. White ${ }^{31}$, A. Wierzcholska ${ }^{35}$, P. Willmann ${ }^{7}$, A. Wörnlein ${ }^{7}$, D. Wouters ${ }^{20}$, M. Zacharias ${ }^{13}$, A. Zajczyk ${ }^{11,33}$, A. A. Zdziarski ${ }^{11}$, A. Zech ${ }^{17}$, and H.-S. Zechlin ${ }^{1}$

(Affiliations can be found after the references)

Received 5 November 2013 / Accepted 18 March 2014

\section{ABSTRACT}

The long gamma-ray burst (GRB) 100621A, at the time the brightest X-ray transient ever detected by Swift-XRT in the $0.3-10 \mathrm{keV}$ range, has been observed with the H.E.S.S. imaging air Cherenkov telescope array, sensitive to gamma radiation in the very-high-energy (VHE, $>100 \mathrm{GeV}$ ) regime. Due to its relatively small redshift of $z \sim 0.5$, the favourable position in the southern sky and the relatively short follow-up time $(<700 \mathrm{~s}$ after the satellite trigger) of the H.E.S.S. observations, this GRB could be within the sensitivity reach of the H.E.S.S. instrument. The analysis of the H.E.S.S. data shows no indication of emission and yields an integral flux upper limit above $\sim 380 \mathrm{GeV}$ of $4.2 \times 10^{-12} \mathrm{~cm}^{-2} \mathrm{~s}^{-1}$ (95\% confidence level), assuming a simple Band function extension model. A comparison to a spectral-temporal model, normalised to the prompt flux at sub-MeV energies, constraints the existence of a temporally extended and strong additional hard power law, as has been observed in the other bright X-ray GRB 130427A. A comparison between the H.E.S.S. upper limit and the contemporaneous energy output in X-rays constrains the ratio between the X-ray and VHE gamma-ray fluxes to be greater than 0.4. This value is an important quantity for modelling the afterglow and can constrain leptonic emission scenarios, where leptons are responsible for the X-ray emission and might produce VHE gamma rays.

Key words. gamma rays: general - gamma-ray burst: individual: GRB 100621A - gamma rays: stars - X-rays: stars

\section{Introduction}

Gamma-ray bursts (GRBs) are brief flashes of X-ray and soft gamma-ray emission traditionally detected in the $\mathrm{keV}$ to $\mathrm{MeV}$

^ Corresponding author: D. Lennarz, e-mail: dirk. lennarz@gatech.edu energy range (for a review see e.g. Gehrels et al. 2009). Their origin and internal acceleration mechanisms are among the most enigmatic questions in contemporary astrophysics. Depending on the length of the prompt gamma-ray emission they are divided into two clases, long and short, if their light curves are longer or shorter than $2 \mathrm{~s}$ respectively. The prompt emission is 
in general well described by a Band function (Band et al. 1993). GRBs exhibit longer-wavelength afterglows that are important for our understanding of the acceleration mechanisms. The emissions are in general consistent with the fireball model (e.g. Piran 1999), where the prompt emission is produced by internal shocks originating in the collision of relativistic jets and the afterglow originates from external shocks, when the fireball ejecta run into the surrounding environment.

Observations at higher energies (HE, above $\sim 20 \mathrm{MeV}$ ) were first made with the Energetic Gamma Ray Experiment Telescope (EGRET). For most detected GRBs the MeV emission is consistent with being a continuation of the GRB spectra at lower energies, without the indication of a cut-off (González et al. 2009). However, one GRB exhibited an additional hard powerlaw component (González et al. 2003), which challenges the interpretation in which the HE emission arises from charged particles through synchrotron radiation. There was an indication of temporally-extended emission, most prominently from GRB 940217, where the emission might have lasted more than 5000 s (Hurley et al. 1994).

Today, the Large Area Telescope (LAT) on board the Fermi Gamma-Ray Space Telescope (Fermi-LAT) observers a subset of very energetic bursts at HE, allowing more detailed studies. For some GRBs (e.g. GRB 080916C, Abdo et al. 2009b) the emission is consistent with a Band function from $\mathrm{keV}$ to $\mathrm{GeV}$ energies, whereas other bursts show an additional hard power-law component at HE (e.g. GRB 090902B \& 090510, Abdo et al. 2009a; Ackermann et al. 2010), which in some cases exhibits a spectral break (e.g. GRB 090926A, Ackermann et al. 2011). These additional spectral components are required for the brightest bursts inside the LAT field of view (Fermi-LAT Collaboration 2013). Additionally, Fermi-LAT finds that the $>100 \mathrm{MeV}$ emission of GRBs starts systematically later than the emission at lower energies, reaching delays up to $40 \mathrm{~s}$ for GRB 090626 and that the duration is also longer than the low-energy equivalent (Fermi-LAT Collaboration 2013). For GRB 130427A, the $\mathrm{HE}$ emission lasted $20 \mathrm{~h}$ and the observations are not in agreement with being synchrotron radiation in the standard afterglow shock model (Ackermann et al. 2014).

GRBs are predicted to emit very-high-energy (VHE, $>100 \mathrm{GeV}$ ) gamma rays in the framework of the fireball model and extending observations of GRBs to the VHE regime is important to further characterise the acceleration and radiation processes at work (e.g. for GRB 130427A, where an inverse Compton scenario has been proposed, see e.g. Tam et al. 2013). Imaging Atmospheric Cherenkov Telescopes (IACTs) such as the High Energy Stereoscopic System (H.E.S.S., see also below), the Major Atmospheric Gamma-Ray Imaging Cherenkov (MAGIC) telescopes and the Very Energetic Radiation Imaging Telescope Array System (VERITAS) are instruments sensitive in this energy range. However, only upper limits on the VHE emission have been reported so far (Aharonian et al. 2009b,a; Albert et al. 2007; Aleksić et al. 2010, 2014; Acciari et al. 2011).

VHE gamma rays are absorbed by interactions with the extragalactic background light (EBL) and can thus only travel limited distances in the Universe (e.g. Dwek \& Krennrich 2013). This poses a severe limitation for GRB observations in this energy range since they typically originate from cosmological distances. However, blazar observations show that the level of EBL extinction is lower than previously thought (e.g. Aharonian et al. 2006b) and it is possible to detect VHE gamma-ray sources even at redshifts above 0.6 with the current generation of telescopes (Furniss et al. 2013).
Its high fluence and very bright afterglow at lower energies identify GRB 100621A as one of the rare and powerful nearby GRBs. Its location within the VHE gamma-ray horizon made this burst a promising target for VHE observations. In this paper, the results of the VHE observations obtained with H.E.S.S. are reported.

\section{GRB 100621A}

GRB 100621A was detected with the Burst Alert Telescope (BAT) on board of the Swift satellite (Barthelmy et al. 2005) on June 21, 2010 at 03:03:32 UT (Ukwatta et al. 2010b), hereafter denoted $t_{0}$. The duration $T_{90}$, the central time interval of $90 \%$ of the prompt flux detected with BAT between $15-350 \mathrm{keV}$, was $(63.6 \pm 1.7) \mathrm{s}$ (Ukwatta et al. 2010a) and the burst was located by Swift's X-ray Telescope (XRT, Burrows et al. 2005) at $\mathrm{RA}(\mathrm{J} 2000)=21^{\mathrm{h}} 01^{\mathrm{m}} 13.12$ and $\operatorname{Dec}(\mathrm{J} 2000)=-51^{\circ} 06^{\prime} 22^{\prime \prime} .5$ with an uncertainty of 1.7 arcsec (radius, $90 \%$ confidence level, Evans et al. 2010). This burst featured an extremely bright X-ray afterglow (Stratta et al. 2010), making it the brightest X-ray transient ever detected by the XRT at that time. Recently, GRB 100621A has been surpassed by GRB 130427A (Maselli et al. 2014).

The Konus-W experiment (Aptekar et al. 1995) on board the WIND spacecraft (Konus-WIND) detected a fluence of GRB $100621 \mathrm{~A}$ in the energy range of $20 \mathrm{keV}-2 \mathrm{MeV}$ of $(3.6 \pm$ $0.4) \times 10^{-5} \mathrm{erg} / \mathrm{cm}^{2}$ within $74 \mathrm{~s}$ after the trigger (Golenetskii et al. 2010). The time-integrated spectrum of the burst is best fit by a Band function (Band et al. 1993), where the low-energy photon index is $-1.69_{-0.07}^{+0.08}$, the high-energy photon index is $-2.46_{-0.45}^{+0.13}$ and the peak energy of the spectral energy distribution is $E_{\mathrm{p}}=95_{-8}^{+9} \mathrm{keV}$ (quoted errors at the $68 \%$ confidence level, Frederiks 2012, priv. comm.). The break energy $E_{0}$ is directly related to $E_{\mathrm{p}}$ via: $E_{0}=E_{\mathrm{p}} /(\alpha+2)$ and the normalisation constant of the Band function can be calculated in such a way that the fluence corresponds to the one measured by Konus-WIND.

The redshift of GRB 100621A has been measured to be $z=0.542$ with the Very Large Telescope (VLT) and the X-shooter spectrograph (Milvang-Jensen et al. 2010). This value was derived from bright emission lines of the host galaxy. The GRB afterglow shows extreme reddening, which is in strong contrast to the blue host galaxy. This suggests that the immediate GRB environment is more dusty than the rest of the host galaxy (Krühler et al. 2011). The optical/near-infrared afterglow exhibits a complex temporal evolution with a steep increase in brightness from around 3.5 to $4.5 \mathrm{ks}$ after the trigger (Krühler et al. 2011).

The GRB position was not visible for the Fermi spacecraft at the time of the Swift trigger due to occultation by the Earth. There is also no LAT coverage of the burst position during the H.E.S.S. observations (see below).

The detection prospects of GRB 100621A in the VHE regime are hard to estimate from the prompt spectrum, because observations carried out by Cherenkov telescopes are typically not contemporaneous with the satellite-based observations, but start on the order of $100 \mathrm{~s}$ later. One can, motivated by the unbroken spectra seen by Fermi-LAT for some bursts and neglecting a possible spectral cut-off and time delay, extrapolate the prompt, time-integrated spectrum measured by Konus-WIND to the VHE regime (Band function extension model). The effect of the absorption on the EBL is estimated using a model by Franceschini et al. (2008), which is interpolated to the GRB redshift. Given the used assumptions 
this extrapolated flux $\left(6.2 \times 10^{-14} \mathrm{~cm}^{-2} \mathrm{~s}^{-1} \mathrm{TeV}^{-1}\right.$ at $\left.1 \mathrm{TeV}\right)$ is in reach of the H.E.S.S. instrument.

The temporal evolution of the Band function extension model flux can be modelled for example as in Gilmore et al. (2013), assuming that the flux in the VHE regime is constant during $T_{90}$ and then decays as a power law $\left(\frac{t}{T_{90}}\right)^{-\gamma}$ when the delay $t$ to the prompt emission grows. This model, consisting of the Band function extension model, EBL absorption and the temporal decay, constitutes the spectral-temporal model used in the analysis, assuming $\gamma=1.5$.

In the spectral-temporal model the flux estimation will be below the reach of the H.E.S.S. instrument for typical observational delays. However, since other bright GRBs seen by Fermi-LAT, like e.g. GRB 130427A, exhibit an additional hard power-law component, one can speculate on temporallyextended and delayed HE emission here. If the component seen in GRB 130427A extended to slightly higher energies than the highest energy photon observed, it would be easily detectable at VHE. It is however unclear, if such a component exists in GRB 100621A, if it extrapolates to the VHE regime and which spectral shape or flux level it should have at the time of observation. Nevertheless, the H.E.S.S. observations provide the ability to detect a possible temporally-extended and strong VHE emission from a hard power-law component.

\section{The High Energy Stereoscopic System}

H.E.S.S. is an array of four IACTs located $1800 \mathrm{~m}$ above sea level in the Khomas Highland of Namibia. It is sensitive to VHE gamma rays between hundreds of $\mathrm{GeV}$ to tens of $\mathrm{TeV}$ by detecting Cherenkov light emitted when the gamma ray is absorbed in the atmosphere in an extensive air shower. Such observations are taken during the parts of the nights without any moon and no clouds in the field of view. Each telescope has a $13 \mathrm{~m}$ diameter and $\sim 100 \mathrm{~m}^{2}$ tessellated mirror surface arranged in a Davies-Cotton design with a focal length of $15 \mathrm{~m}$. The telescopes are arranged in a square with $120 \mathrm{~m}$ side length with one diagonal oriented north-south. Furthermore, each telescope is equipped with a pixelated camera of 960 photomultiplier tubes (PMTs) with Winston cones in front to improve the light collection efficiency. One pixel subtends approximately $0.16^{\circ}$, resulting in a total field of view of $5^{\circ}$ in diameter. The triggering is done in three different stages: at PMT level, at telescope and at array level (Funk et al. 2004). Only events recorded by at least two of the four telescopes are used, allowing stereoscopic image analysis. This results in an angular resolution (68\% containment) of typically $0.1^{\circ}$ and an energy resolution of $\sim 15 \%$. The H.E.S.S. effective area and energy threshold are largely influenced by the zenith angle of the observation, leading to a higher energy threshold the larger the zenith angle of the observation. A more comprehensive summary of H.E.S.S. can be found in Aharonian et al. (2006a) and the references therein.

\section{Data collection and analysis}

In order to allow rapid follow-up observations, the H.E.S.S. data acquisition system is connected to the GRB Coordinates Network $(\mathrm{GCN})^{1}$. Notices of GRBs detected by satellites are received via socket connection and automatically processed on site. Currently, H.E.S.S. accepts notices from Swift-BAT and Fermi-LAT as triggers if they have a positional uncertainty $<2.5^{\circ}$

\footnotetext{
http://gcn.gscfc.nasa.gov
}

and more detailed trigger conditions are met e.g. the significance, a position incompatible with known sources and the quality of the trigger data. Observations should be started immediately by the observers present at the telescopes if the trigger is received during dark time (i.e. night and no moon) with fair weather conditions and if the GRB position can be observed with a zenith angle smaller than $45^{\circ}$ to ensure a reasonably low energy threshold. Recently, this human-in-the-loop process has been replaced by a fully automated repointing procedure, which was however not yet present at the observation of GRB 100621A. Further technical details of the H.E.S.S. GRB programme can be found in Lennarz et al. (2013).

The trigger for GRB 100621A from Swift-BAT was received in Namibia at 03:04:01 UT, which is $29 \mathrm{~s}$ after $t_{0}$. However, due to technical problems, observations were started only at 03:14:55 UT which is $683 \mathrm{~s}$ after $t_{0}$. Due to moonrise only two observations with a nominal duration of $28 \mathrm{~min}$ were taken. The burst was observed in "wobble mode" in which the observation position is displaced from the centre of the camera (Fomin et al. 1994) to allow for observation and background estimation from the same field of view (reflected-regionbackground model, see Berge et al. 2007). The first observation was displaced by $-0.5^{\circ}$ in declination and started at a zenith angle of $31.7^{\circ}$, reaching a final position of $34.6^{\circ}$ (mean value of $32.7^{\circ}$ ) with a deadtime-corrected livetime of $1576 \mathrm{~s}$. For the second observation (displaced $0.5^{\circ}$ in declination), the zenith angle range was $34.1-37.3^{\circ}$ (mean value of $36.1^{\circ}$ ). It started at 03:45:23 UT and had a livetime of $1574 \mathrm{~s}$. All data were taken during good weather conditions with good hardware status of all four telescopes.

The data calibration, image cleaning, Hillas moment calculation (Hillas 1996) and event reconstruction is done as described in Aharonian et al. (2006a) with the standard H.E.S.S. analysis software $^{2}$. In this reference, three different selection cuts (standard, hard, loose) to reject background caused by cosmic-ray showers are described, suited for different source scenarios. The background rejection can also be done with a multivariate cut using a decision tree obtained from a boosting algorithm (boosted decision tree, for details see Ohm et al. 2009). Recently, selection cuts corresponding to the loose cuts from Aharonian et al. (2006a) have been added to the multivariate analysis. A sizecut of 40 photo electrons, a $\theta^{2}$-cut of 0.02 degrees $^{2}$ (where $\theta$ is the angular distance between the reconstructed event direction and the assumed source position), and a $\zeta$-cut of 0.85 (where $\zeta$ denotes the classifier of the boosted decision tree, see Ohm et al. 2009) are used. Due to EBL absorption, the spectrum of GRB $100621 \mathrm{~A}$ is expected to be very soft, which makes the sensitivity of the analysis highly dependent on the energy threshold. The lower intensity cut of loose cuts reduces the energy threshold compared to standard and hard cuts. Thus, the multivariate loose cuts have the highest sensitivity and are used in this analysis.

After applying the selection cuts, the number of events $\left(N_{\text {on }}\right)$ in the signal region ("on-region") around the GRB position and the number of events $\left(N_{\text {off }}\right)$ in the regions used to estimate the background ("off-regions") can be used to calculate the significance of the gamma-ray excess using Eq. (17) of $\mathrm{Li} \& \mathrm{Ma}$ (1983). A normalisation factor $\alpha$ is applied to correct for the different number of on- and off-regions.

The energy threshold for the spectral analysis, $E_{\mathrm{th}}=$ $383 \mathrm{GeV}$, is defined by the energy below which the energy bias becomes larger than $10 \%$. This approach is conservative,

2 Version hap-11-07-pl01. 
Table 1. Results of the search for excess photons.

\begin{tabular}{llllll}
\hline \hline & $N_{\text {on }}$ & $N_{\text {off }}$ & $\alpha$ & $N_{\text {excess }}$ & Significance \\
\hline Total & 46 & 427 & 0.118 & $-4_{-7}^{+8}$ & -0.6 \\
First 300 s & 8 & 39 & 0.125 & $3_{-3}^{+3}$ & 1.2 \\
1st observation & 26 & 197 & 0.125 & $1_{-5}^{+6}$ & 0.3 \\
2nd observation & 20 & 230 & 0.111 & $-6_{-5}^{+5}$ & -1.1 \\
\hline
\end{tabular}

Notes. $N_{\text {on }}$ is the number of gamma-ray candidates in the signal region around the GRB position and $N_{\text {off }}$ the background estimate. When scaled by the normalisation factor $\alpha$ they yield the number of excess events $N_{\text {excess }}=N_{\text {on }}-\alpha N_{\text {off }}$.

Table 2. Integral flux upper limits.

\begin{tabular}{lccc}
\hline \hline & Above $E_{\mathrm{th}}{ }^{a}$ & \multicolumn{2}{c}{ Differential $^{b}$ at } \\
\hline & & $E_{\text {th }}$ & $1 \mathrm{TeV}$ \\
\hline Total & $4.2 \times 10^{-12}$ & $6.1 \times 10^{-11}$ & $1.0 \times 10^{-13}$ \\
1st observation & $6.4 \times 10^{-12}$ & $9.4 \times 10^{-11}$ & $1.5 \times 10^{-13}$ \\
2nd observation & $3.8 \times 10^{-12}$ & $5.3 \times 10^{-11}$ & $1.6 \times 10^{-13}$ \\
\hline
\end{tabular}

Notes. Upper limits correspond to a confidence level of $95 \%$ as derived from the H.E.S.S. spectral analysis, assuming the EBL absorbed simple Band function extension model. For the first observation and the total data set the energy threshold is $E_{\text {th }}=383 \mathrm{GeV}$ and for the second observation $E_{\mathrm{th}}=422 \mathrm{GeV}$. The integral upper limits are also expressed as a differential flux at certain energies. ${ }^{(a)}$ Units $\mathrm{cm}^{-2} \mathrm{~s}^{-1}$. (b) Units $\mathrm{cm}^{-2} \mathrm{~s}^{-1} \mathrm{TeV}^{-1}$.

because it reduces systematic uncertainties in the estimation of the effective area. H.E.S.S. can still detect gamma rays with energies below this value and all events are used when estimating the significance. However, the spectral analysis is restricted to events with reconstructed energies above the energy threshold.

\section{Results}

The results of the analysis of the H.E.S.S. data taken for GRB 100621 A are shown in Table 1. No excess is observed using the total data set. In order to search for emission on shorter time scales and closer to $t_{0}$ a further analysis was done on each observation separately and on the events corresponding to the first $300 \mathrm{~s}$ of the first observation. Shorter time scales are not possible because the number of events in the on-region would become too low to estimate the significance. No significant excess is found here either. The result for the total dataset has also been crosschecked with an independent calibration and analysis of the data (Becherini et al. 2011).

Upper limits on the number of excess events are calculated using the method of Rolke et al. (2005). These upper limits are converted to integral flux upper limits using the H.E.S.S. effective area. The spectral shape is assumed to follow the Band function extension model plus EBL absorption (a temporal component plays no roll in the calculation). The integral limit can be presented as a differential flux on the assumed spectrum of $1.0 \times 10^{-13} \mathrm{~cm}^{-2} \mathrm{~s}^{-1} \mathrm{TeV}^{-1}$ at $1 \mathrm{TeV}$ at $95 \%$ confidence level (see Table 2).

Figure 1 shows a graphical representation of the upper limit and compares it to the spectral-temporal model. It can also be seen that the spectral shape in the H.E.S.S. energy range is mostly dominated by the EBL absorption. Thus, changing the spectral model from the Band function extension model to e.g. an $E^{-2}$ spectrum would change the limits only marginally.

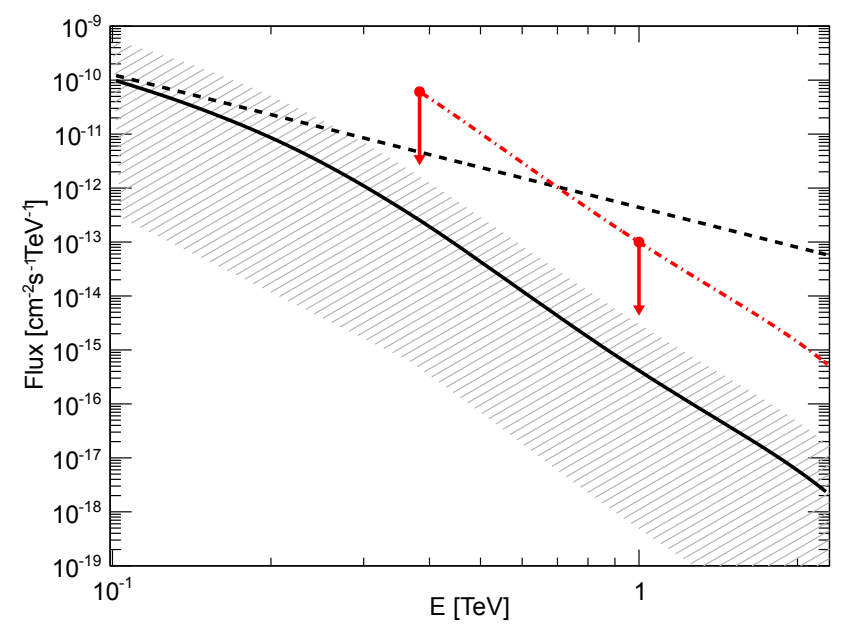

Fig. 1. Solid line: spectral-temporal model matching the H.E.S.S. observation window, dashed line: same spectrum without applying the EBL model by Franceschini et al. (2008). It can be seen that the spectral shape is dominated by the EBL absorption in the H.E.S.S. energy range. The red dashed-dotted line shows the spectrum that corresponds to the limits given in Table 2 as obtained by the analysis of the total data set, where the red dots are the two given differential representations. The shaded area shows the effect of varying the Konus-WIND high-energy photon index $\beta$ within its one-sigma error.

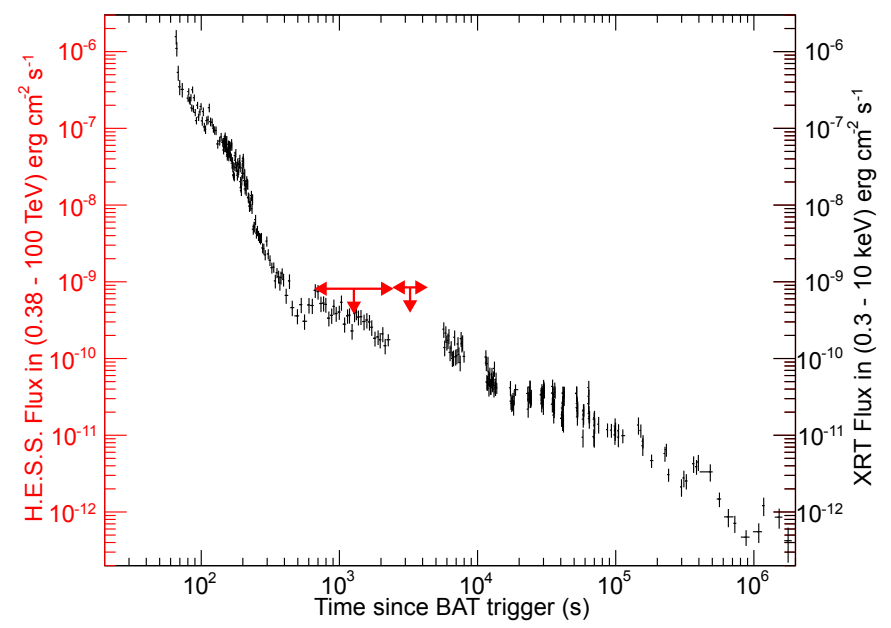

Fig. 2. Comparison of the VHE upper limits (95\% confidence level) on the energy output above the energy threshold (in lighter colour) using the Band function extension model (no EBL correction applied) with the XRT energy flux (in darker colour, de-absorbed, from the Swift Burst Analyser, Evans et al. 2009, 2007). Horizontal arrows indicate the start and end time of the observations from which the corresponding upper limit is derived.

Changing the decay factor $\gamma$ in the temporal decay e.g. to 1.0 would move the model up by a factor of $\sim 5$, which is small compared to the other uncertainties of the extrapolation. This decay index has been observed by Fermi-LAT, however the characteristic time scale is the time of the LAT peak emission (Fermi-LAT Collaboration 2013) and its relation to the $T_{90}$ at lower energies remains unclear.

In Fig. 2 the energy output after correcting for absorption effects in the H.E.S.S. $(0.38-100 \mathrm{TeV})$ and XRT energy range $(0.3-10 \mathrm{keV})$ is compared. As can be seen, GRB $100621 \mathrm{~A}$ exhibited an extremely bright X-ray afterglow at earlier times. The H.E.S.S. observations were obtained during the shallow X-ray phase and do not cover the steep increase in brightness in the 
optical/near-infrared afterglow. During the first observation the ratio between the energy output (using the energy ranges given in Fig. 2$)$ in X-ray $\left(F_{\mathrm{X} \text {-ray }}\right)$ and $\operatorname{VHE}\left(F_{\mathrm{VHE}}\right)$ can be constrained to be $\frac{F_{\mathrm{X}-\mathrm{ray}}}{F_{\mathrm{VHE}}}>0.4$ at $95 \%$ confidence level.

\section{Interpretation}

Figure 1 shows that the H.E.S.S. upper limit is above the simple temporal Band function extension model. Furthermore, to illustrate the large uncertainty that comes with the Band function extension model the Konus-WIND high-energy photon index is varied within its one-sigma error. This gives an uncertainty of several orders of magnitude, without taking into account the errors of the other model parameters and their correlations with $\beta$. Thus, the H.E.S.S. upper limit is not able to exclude the simple temporal Band function extension model.

A temporally-extended and additional hard power law with an $E^{-2}$ spectrum would have been detected by H.E.S.S. if its unabsorbed fluence between $383 \mathrm{GeV}$ and $10 \mathrm{TeV}$ is in excess of $\sim 3 \times 10^{-6} \mathrm{erg} \mathrm{cm}^{-2}$. The contribution from the temporal Band function extension model is small at the time of the H.E.S.S. observations and can be neglected. This fluence limit is within a factor of about \pm 2 independent of the power law index (between -1.5 and -2.5$)$, because the spectral shape in the H.E.S.S. energy range is dominated by the imprint of the EBL absorption.

Variations of the spectral index have however a strong influence on the fluence at lower energies. The above limit (for $E^{-2}$ ) corresponds to $1 \times 10^{-5} \mathrm{erg} \mathrm{cm} \mathrm{cm}^{-2}$ or $2 \times 10^{-6} \mathrm{erg} \mathrm{cm}^{-2}$ between $10 \mathrm{keV}$ and $10 \mathrm{GeV}$ and $>100 \mathrm{MeV}$ respectively, while for $E^{-1.5}$ it changes to $2 \times 10^{-7} \mathrm{erg} \mathrm{cm}^{-2}$ and $4 \times 10^{-8} \mathrm{erg} \mathrm{cm}^{-2}$. For GRB 130427A, the LAT measured a $>100 \mathrm{MeV}$ fluence in the $100 \mathrm{ks}$ following the trigger of $(7 \pm 1) \times 10^{-4} \mathrm{erg} \mathrm{cm}^{-2}$ with a typical spectral index of $E^{-2}$ (Ackermann et al. 2014). The presence of a component that strong during the afterglow phase of GRB 100621A can be excluded, which is remarkable, given that both GRBs were of similar brightness in X-rays.

Motivated by the temporal model discussed earlier, one can assume that the fluence during the H.E.S.S. observations is at the $1 \%$ level compared to the prompt phase. Thus, an additional component as strong as in GRB 090902B $\left(1.007_{-0.057}^{+0.059} \times\right.$ $10^{-4} \mathrm{erg} \mathrm{cm}^{-2}$ between $10 \mathrm{keV}$ and $10 \mathrm{GeV}$, obtained during the time of the first LAT photon and the GBM $T_{95}$, Fermi-LAT Collaboration 2013) is excluded if it had a spectrum following $E^{-1.5}$ at the time of the H.E.S.S. observations. Both interpretations are subjected to the assumption of no spectral break in the extra component.

In a leptonic scenario, the X-ray afterglow is typically modelled as electron synchrotron emission in the external shock. The accelerated electrons could upscatter photons generated e.g. by synchrotron emission from the same population of electrons via the inverse Compton process (synchrotron self-Compton, SSC), which would lead to VHE radiation. In most modeles the energy outputs in X-rays and VHE gamma radiation are proportional. Thus the upper limits on the energy output obtained here can be used to constrain such modelling.

\section{Summary}

In this paper, the analysis of the H.E.S.S. data on GRB 100621A is presented. A significant excess has neither been observed in the total data set, nor on shorter time scales closer to the prompt emission. This constrains the possibility of a temporallyextended emission in the form of an additional hard power law like it has been observed by Fermi-LAT in previous bright bursts. A component as strong as in GRB 130427A is not compatible with the H.E.S.S. measurements.

GRB 100621A is one of the brightest X-ray sources detected by Swift with a very bright X-ray afterglow. The H.E.S.S. observations started during the shallow decline of the X-ray light curve and the upper limits on the energy output during that time are comparable to the level of the X-ray emission. The ratio between the X-ray and VHE flux is constrained to be greater than 0.4 , which can constrain the synchrotron modelling of the afterglow due to the apparent lack of detected inverse Compton emission.

The advent of H.E.S.S. II, which is the world's largest Imaging Atmospheric Cherenkov Telescope, significantly enhances the chances of a VHE GRB detection. The telescope will have a lower energy threshold (tens of $\mathrm{GeV}$ ) and a higher performance drive system that will reduce the response time to a GRB alert (Hofverberg \& H.E.S.S. Collaboration 2011).

Acknowledgements. The support of the Namibian authorities and of the University of Namibia in facilitating the construction and operation of H.E.S.S. is gratefully acknowledged, as is the support by the German Ministry for Education and Research (BMBF), the Max Planck Society, the German Research Foundation (DFG), the French Ministry for Research, the CNRS-IN2P3 and the Astroparticle Interdisciplinary Programme of the CNRS, the UK Science and Technology Facilities Council (STFC), the IPNP of the Charles University, the Czech Science Foundation, the Polish Ministry of Science and Higher Education, the South African Department of Science and Technology and National Research Foundation, and by the University of Namibia. We appreciate the excellent work of the technical support staff in Berlin, Durham, Hamburg, Heidelberg, Palaiseau, Paris, Saclay, and in Namibia in the construction and operation of the equipment.

\section{References}

Abdo, A. A., Ackermann, M., Ajello, M., et al. 2009a, ApJ, 706, L138 Abdo, A. A., Ackermann, M., Arimoto, M., et al. 2009b, Science, 323, 1688 Acciari, V. A., Aliu, E., Arlen, T., et al. 2011, ApJ, 743, 62

Ackermann, M., Asano, K., Atwood, W. B., et al. 2010, ApJ, 716, 1178

Ackermann, M., Ajello, M., Asano, K., et al. 2011, ApJ, 729, 114

Ackermann, M., Ajello, M., Asano, K., et al. 2014, Science, 343, 42

Aharonian, F., Akhperjanian, A. G., Bazer-Bachi, A. R., et al. 2006a, A\&A, 457, 899

Aharonian, F., Akhperjanian, A. G., Bazer-Bachi, A. R., et al. 2006b, Nature, 440, 1018

Aharonian, F., Akhperjanian, A. G., Barres de Almeida, U., et al. 2009a, ApJ, 690,1068

Aharonian, F., Akhperjanian, A. G., Barres de Almeida, U., et al. 2009b, A\&A, 495,505

Albert, J., Aliu, E., Anderhub, H., et al. 2007, ApJ, 667, 358

Aleksić, J., Anderhub, H., Antonelli, L. A., et al. 2010, A\&A, 517, A5

Aleksić, J., Ansoldi, S., Antonelli, L. A., et al. 2014, MNRAS, 437, 3103

Aptekar, R. L., Frederiks, D. D., Golenetskii, S. V., et al. 1995, Space Sci. Rev., 71,265

Band, D., Matteson, J., Ford, L., et al. 1993, ApJ, 413, 281

Barthelmy, S. D., Barbier, L. M., Cummings, J. R., et al. 2005, Space Sci. Rev., 120,143

Becherini, Y., Djannati-Ataï, A., Marandon, V., Punch, M., \& Pita, S. 2011, Astropart. Phys., 34, 858

Berge, D., Funk, S., \& Hinton, J. 2007, A\&A, 466, 1219

Burrows, D. N., Hill, J. E., Nousek, J. A., et al. 2005, Space Sci. Rev., 120, 165

Dwek, E., \& Krennrich, F. 2013, Astropart. Phys., 43, 112

Evans, P. A., Beardmore, A. P., Page, K. L., et al. 2007, A\&A, 469, 379

Evans, P. A., Beardmore, A. P., Page, K. L., et al. 2009, MNRAS, 397, 1177

Evans, P. A., Goad, M. R., Osborne, J. P., \& Beardmore, A. P. 2010, GRB Coordinates Network, 10873, 1

Fermi-LAT Collaboration. 2013, ApJS, submitted [arXiv: 1303.2908]

Fomin, V. P., Stepanian, A. A., Lamb, R. C., et al. 1994, Astropart. Phys., 2, 137

Franceschini, A., Rodighiero, G., \& Vaccari, M. 2008, A\&A, 487, 837

Funk, S., Hermann, G., Hinton, J., et al. 2004, Astropart. Phys., 22, 285

Furniss, A., Williams, D. A., Danforth, C., et al. 2013, ApJ, 768, L31

Gehrels, N., Ramirez-Ruiz, E., \& Fox, D. B. 2009, ARA\&A, 47, 567 
Gilmore, R. C., Bouvier, A., Connaughton, V., et al. 2013, Exp. Astron., 35, 413 Golenetskii, S., Aptekar, R., Frederiks, D., et al. 2010, GRB Coordinates Network, 10882, 1

González, M. M., Dingus, B. L., Kaneko, Y., et al. 2003, Nature, 424, 749

González, M. M., Carrillo-Barragán, M., Dingus, B. L., et al. 2009, ApJ, 696, 2155

Hillas, A. M. 1996, Space Sci. Rev., 75, 17

Hofverberg, P., \& H.E.S.S. Collaboration 2011, Nucl. Instrum. Methods Phys. Res. A, 639, 23

Hurley, K., Dingus, B. L., Mukherjee, R., et al. 1994, Nature, 372, 652

Krühler, T., Greiner, J., Schady, P., et al. 2011, A\&A, 534, A108

Lennarz, D., Chadwick, P. M., Domainko, W., et al. 2013, in Proc. 7th Huntsville Gamma-Ray Burst Symposium, Nashville, Tennessee, USA, eds. N. Gehrels,

M. S. Briggs, \& V. Connaughton, eConf C1304143, 38 [arXiv: 1307.6897]

Li, T.-P., \& Ma, Y.-Q. 1983, ApJ, 272, 317

Maselli, A., Melandri, A., Nava, L., et al. 2014, Science, 343, 48

Milvang-Jensen, B., Goldoni, P., Tanvir, N. R., et al. 2010, GRB Coordinates Network, 10876, 1

Ohm, S., van Eldik, C., \& Egberts, K. 2009, Astropart. Phys., 31, 383

Piran, T. 1999, Phys. Rep., 314, 575

Rolke, W. A., López, A. M., \& Conrad, J. 2005, Nucl. Instrum. Methods Phys. Res. A, 551, 493

Stratta, G., D'Elia, V., \& Ukwatta, T. N. 2010, GRB Coordinates Network, 10877,1

Tam, P.-H. T., Tang, Q.-W., Hou, S.-J., Liu, R.-Y., \& Wang, X.-Y. 2013, ApJ, 771, L13

Ukwatta, T. N., Barthelmy, S. D., Baumgartner, W. H., et al. 2010a, GRB Coordinates Network, 10875, 1

Ukwatta, T. N., Barthelmy, S. D., Baumgartner, W. H., et al. 2010b, GRB Coordinates Network, 10870, 1

1 Universität Hamburg, Institut für Experimentalphysik, Luruper Chaussee 149, 22761 Hamburg, Germany

2 Max-Planck-Institut für Kernphysik, PO Box 103980, 69029 Heidelberg, Germany

e-mail: dirk. lennarz@gatech.edu

3 Dublin Institute for Advanced Studies, 31 Fitzwilliam Place, Dublin 2, Ireland

4 National Academy of Sciences of the Republic of Armenia, Yerevan, Armenia

5 Yerevan Physics Institute, 2 Alikhanian Brothers St., 375036 Yerevan, Armenia

6 Institut für Physik, Humboldt-Universität zu Berlin, Newtonstr. 15, 12489 Berlin, Germany

7 Universität Erlangen-Nürnberg, Physikalisches Institut, ErwinRommel-Str. 1, 91058 Erlangen, Germany

8 University of Durham, Department of Physics, South Road, Durham DH1 3LE, UK

9 DESY, 15735 Zeuthen, Germany

10 Institut für Physik und Astronomie, Universität Potsdam, KarlLiebknecht-Strasse 24/25, 14476 Potsdam, Germany

11 Nicolaus Copernicus Astronomical Center, ul. Bartycka 18, 00-716 Warsaw, Poland

12 Landessternwarte, Universität Heidelberg, Königstuhl, 69117 Heidelberg, Germany

13 Institut für Theoretische Physik, Lehrstuhl IV: Weltraum und Astrophysik, Ruhr-Universität Bochum, 44780 Bochum, Germany

14 Institut für Astro- und Teilchenphysik, Leopold-FranzensUniversität Innsbruck, 6020 Innsbruck, Austria
15 Laboratoire Leprince-Ringuet, École Polytechnique, CNRS/IN2P3, 91128 Palaiseau, France

16 Unit for Space Physics, North-West University, 2520 Potchefstroom, South Africa

17 LUTH, Observatoire de Paris, CNRS, Université Paris Diderot, 5 place Jules Janssen, 92190 Meudon, France

18 LPNHE, Université Pierre et Marie Curie Paris 6, Université Denis Diderot Paris 7, CNRS/IN2P3, 4 place Jussieu, 75252 Paris Cedex 5, France

19 Institut für Astronomie und Astrophysik, Universität Tübingen, Sand 1, 72076 Tübingen, Germany

20 DSM/Irfu, CEA Saclay, 91191 Gif-Sur-Yvette Cedex, France

21 Astronomical Observatory, The University of Warsaw, Al. Ujazdowskie 4, 00-478 Warsaw, Poland

22 now at Harvard-Smithsonian Center for Astrophysics, 60 garden Street, Cambridge MA, 02138, USA

23 School of Physics, University of the Witwatersrand, 1 Jan Smuts Avenue, Braamfontein, 2050 Johannesburg, South Africa

24 Oskar Klein Centre, Department of Physics, Stockholm University, Albanova University Center, 10691 Stockholm, Sweden

25 Université Bordeaux 1, CNRS/IN2P3, Centre d'Études Nucléaires de Bordeaux Gradignan, 33175 Gradignan, France

26 Funded by contract ERC-StG-259391 from the European Community,

27 University of Namibia, Department of Physics, 13301 Private Bag, Windhoek, Namibia

28 School of Chemistry \& Physics, University of Adelaide, 5005 Adelaide, Australia

29 APC, AstroParticule et Cosmologie, Université Paris Diderot, CNRS/IN2P3, CEA/Irfu, Observatoire de Paris, Sorbonne Paris Cité, 10 rue Alice Domon et Léonie Duquet, 75205 Paris Cedex 13, France,

30 UJF-Grenoble 1/CNRS-INSU, Institut de Planétologie et d'Astrophysique de Grenoble (IPAG) UMR 5274, 38041 Grenoble, France

31 Department of Physics and Astronomy, The University of Leicester, University Road, Leicester, LE1 7RH, UK

32 Instytut Fizyki Jạdrowej PAN, ul. Radzikowskiego 152, 31-342 Kraków, Poland

33 Laboratoire Univers et Particules de Montpellier, Université Montpellier 2, CNRS/IN2P3, CC 72, Place Eugène Bataillon, 34095 Montpellier Cedex 5, France

34 Laboratoire d'Annecy-le-Vieux de Physique des Particules, Université de Savoie, CNRS/IN2P3, 74941 Annecy-le-Vieux, France

35 Obserwatorium Astronomiczne, Uniwersytet Jagielloński, ul. Orla 171, 30-244 Kraków, Poland

36 Toruń Centre for Astronomy, Nicolaus Copernicus University, ul. Gagarina 11, 87-100 Torun, Poland

37 School of Physics \& Astronomy, University of Leeds, Leeds LS2 9JT, UK

38 Charles University, Faculty of Mathematics and Physics, Institute of Particle and Nuclear Physics, V Holešovičkách 2, 18000 Prague 8 , Czech Republic

39 Institute of Astronomy and Department of Physics, National Tsing Hua University, 30013 Hsinchu, Taiwan 